\title{
Erratum
}

\section{Parallelizability of Homogeneous Spaces, II}

\author{
W. Singhof and D. Wemmer
}

Fachbereich Mathematik, Universität, Erwin-Schrödinger-Strasse, D-6750 Kaiserslautern, Federal Republic of Germany

Math. Ann. 274, 157 (1986)

In the list of stably parallelizable quotients [2, Theorem 2] case (vi) is wrongly stated. It should read:

(vi) $\operatorname{Sp}(n) /(S U(2) \times \ldots \times S U(2))=Z_{n, k}$ where $k$ denotes the number of factors $S U(2)$, with $2 k \leqq n$.

As a consequence, Theorem 1 and the ensuing discussion require the additional hypothesis that $G$ is not isomorphic to $\operatorname{Sp}(n), n \geqq 4$. In the general case, we have instead:

Theorem 1*. Let $G$ be a simple 1-connected compact Lie group and $H$ a closed connected subgroup. Denote by I the ideal of $R O(H)$ which is generated by the elements

$$
\begin{aligned}
& (\psi \mid H-\operatorname{dim} \psi) \quad \text { where } \psi \in R O(G) \text {, and } \\
& \varphi \cdot\left(\lambda \mid H-\operatorname{dim}_{\mathbb{C}} \lambda\right) \quad \text { where } \varphi \in R S p(H), \lambda \in R S p(G) .
\end{aligned}
$$

Then $G / H$ is stably parallelizable if and only if

$$
\left(\operatorname{Ad}_{H}-\operatorname{dim} H\right) \in I \text {. }
$$

The error comes from Sect. 7 which should be replaced by the following text:

Observe that the tensor product of two symplectic representations is real. Therefore, if $H$ is a closed subgroup of any compact Lie group $G$, we have a natural homomorphism

$$
\bar{\alpha}: R O(H) \otimes_{R O(G) \oplus R S_{p(H)} \cdot \tilde{R} \tilde{S}_{p(G)}} \mathbb{Z} \rightarrow K O(G / H) .
$$

Here, $R \widetilde{R} p(G)$ denotes the module of all virtual symplectic representations of dimension 0 .

$(7.3)^{*}$ Corollary. The manifolds $Z_{n, k}=\mathrm{Sp}(n) /(S U(2))^{k}$ are parallelizable. 
Proof. Write $R(\mathrm{Sp}(n))=\mathbb{Z}\left[\lambda_{1}, \ldots, \lambda_{n}\right]$ where $\lambda_{i}$ are the fundamental representations, with $\lambda_{2 i}$ orthogonal and $\lambda_{2 i+1}$ symplectic; $R\left(S U(2)^{k}\right)=\mathbb{Z}\left[\varphi_{1}, \ldots, \varphi_{n}\right]$ where $\varphi_{i}$ is the projection onto the $i$-th factor. Writing $H=S U(2)^{k}$, we have

$$
\begin{aligned}
\operatorname{Ad}_{\boldsymbol{H}} & =\sum_{i=1}^{k}\left(\varphi_{i}^{2}-1\right) \\
& =\left(\sum \varphi_{i}\right) \cdot\left(\lambda_{1} \mid H-2 n\right)+\lambda_{2}\left|H+(2 n-2 k) \cdot 2 \lambda_{1}\right| H+l, l \in \mathbb{Z} .
\end{aligned}
$$

As $\varphi_{i}$ is symplectic and $2 \lambda_{1}$ is real $\mathrm{Sp}(n) / H$ is stably parallelizable by (7.1)*. To obtain parallelizability, observe that there is a free $\mathbb{Z}_{2} \times \mathbb{Z}_{2}$-operation on $Z_{n, k}$ for $k \geqq 2$ (the case $k=1$ has been dealt with in $[1,4.5]$ ):

Let $R$ be the multiplication by $\operatorname{diag}(i, i, 1, \ldots 1, \ldots, 1)$ and $S$ by $\operatorname{diag}(1,1, i, i, 1, \ldots, 1)$ from the right. The group generated by $R$ and $S$ gives the desired action.

(7.4)* Lemma. Let $H$ be a subgroup of $\mathrm{Sp}(n)$ such that $\mathrm{SU}(2 n) / H$ is stably parallelizable. Then $\mathrm{Sp}(n) / H$ is diffeomorphic to $\mathrm{Sp}(2) / S U(2)$ or $\mathrm{Sp}(n) /(\mathrm{Sp}(1) \times \ldots \times \mathrm{Sp}(1))$.

Proof. This follows from $[2,6.5]$.

$(7.5)^{*}$ Lemma. Let $H$ be a subgroup of $\mathrm{Sp}(n)$ such that $\mathrm{Sp}(n) / H$ is stably parallelizable, but $S U(2 n) / H$ is not. Then $\mathrm{Sp}(n) / H$ is diffeomorphic to $Z_{n, k}$ or to $\mathrm{Sp}(n) / \mathrm{Sp}(k)$.

This follows by considering Pontrjagin and Stiefel-Whitney classes.

Finally $[2,7.6]$ remains unchanged.

\section{References}

1. Singhof, W.: Parallelizability of homogeneous spaces. I. Math. Ann. 260, 101-116 (1982)

2. Singhof, W., Wemmer, D.: Parallelizability of homogeneous spaces. II. Math. Ann. 274, 157-176 (1986)

Received November 4, 1986 